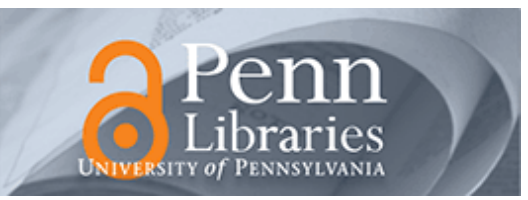

University of Pennsylvania

ScholarlyCommons

10-24-2008

\title{
Two-dimensional photonic crystals with anisotropic unit cells imprinted from poly(dimethylsiloxane) membranes under elastic deformation
}

\author{
Xuelian Zhu \\ University of Pennsylvania, xuelian@seas.upenn.edu \\ Ying Zhang \\ University of Pennsylvania, ygzhang@seas.upenn.edu \\ Dinesh Chandra \\ University of Pennsylvania, chandrad@seas.upenn.edu \\ Shih-Chieh Cheng \\ University of Pennsylvania, chengsh@seas.upenn.edu \\ James M. Kikkawa \\ University of Pennsylvania, kikkawa@physics.upenn.edu \\ Follow this and additional works at: https://repository.upenn.edu/mse_papers
}

\section{See next page for additional authors}

Recommended Citation

Zhu, X., Zhang, Y., Chandra, D., Cheng, S., Kikkawa, J. M., \& Yang, S. (2008). Two-dimensional photonic crystals with anisotropic unit cells imprinted from poly(dimethylsiloxane) membranes under elastic deformation. Retrieved from https://repository.upenn.edu/mse_papers/167

Copyright 2008 American Institute of Physics. This article may be downloaded for personal use only. Any other use requires prior permission of the author and the American Institute of Physics.

Reprinted from:

Two-dimensional photonic crystals with anisotropic unit cells imprinted from poly(dimethylsiloxane) membranes under elastic deformation Xuelian Zhu, Ying Zhang, Dinesh Chandra, Shih-Chieh Cheng, James M. Kikkawa, and Shu Yang, Appl. Phys. Lett. 93, 161911 (2008), DOI:10.1063/1.3006061

Publisher URL: http://link.aip.org/link/?APPLAB/93/161911/1

This paper is posted at ScholarlyCommons. https://repository.upenn.edu/mse_papers/167

For more information, please contact repository@pobox.upenn.edu. 


\title{
Two-dimensional photonic crystals with anisotropic unit cells imprinted from poly(dimethylsiloxane) membranes under elastic deformation
}

\author{
Abstract \\ We study structural symmetries of two-dimensional (2D) photonic crystals with anisotropic unit cells, \\ including square- and rectangular-lattices with orientationally modulated elliptic motifs, and a compound \\ structure consisting of circles with sixfold rotational symmetry and elliptical lines with twofold symmetry, \\ which are created through elastic deformation of a single elastomeric membrane with circular pores. We \\ then investigate the photonic bandgap (PBG) properties of the corresponding 2D Si posts and their \\ tolerance to the structural deviation. We find that in the compound structure the overall PBGs are \\ dominated by the sublattice with a higher symmetry, while the total symmetry is determined by the one \\ with a lower symmetry.

\section{Keywords} \\ DESIGN, LIGHT

\section{Comments} \\ Copyright 2008 American Institute of Physics. This article may be downloaded for personal use only. Any \\ other use requires prior permission of the author and the American Institute of Physics. \\ Reprinted from: \\ Two-dimensional photonic crystals with anisotropic unit cells imprinted from poly(dimethylsiloxane) \\ membranes under elastic deformation Xuelian Zhu, Ying Zhang, Dinesh Chandra, Shih-Chieh Cheng, \\ James M. Kikkawa, and Shu Yang, Appl. Phys. Lett. 93, 161911 (2008), DOI:10.1063/1.3006061 \\ Publisher URL: http://link.aip.org/link/?APPLAB/93/161911/1
}

Author(s)

Xuelian Zhu, Ying Zhang, Dinesh Chandra, Shih-Chieh Cheng, James M. Kikkawa, and Shu Yang 


\title{
Two-dimensional photonic crystals with anisotropic unit cells imprinted from poly(dimethylsiloxane) membranes under elastic deformation
}

\author{
Xuelian Zhu, ${ }^{1}$ Ying Zhang, ${ }^{1}$ Dinesh Chandra, ${ }^{1}$ Shih-Chieh Cheng, ${ }^{1}$ James M. Kikkawa, ${ }^{2}$ \\ and Shu Yang ${ }^{1, a)}$ \\ ${ }_{1}^{1}$ Department of Materials Science and Engineering, University of Pennsylvania, 3231 Walnut Street, \\ Philadelphia, Pennsylvania 19104, USA \\ ${ }^{2}$ Department of Physics and Astronomy, University of Pennsylvania, 209 South 33rd Street, Philadelphia, \\ Pennsylvania 19104, USA
}

(Received 20 August 2008; accepted 1 October 2008; published online 24 October 2008)

\begin{abstract}
We study structural symmetries of two-dimensional (2D) photonic crystals with anisotropic unit cells, including square- and rectangular-lattices with orientationally modulated elliptic motifs, and a compound structure consisting of circles with sixfold rotational symmetry and elliptical lines with twofold symmetry, which are created through elastic deformation of a single elastomeric membrane with circular pores. We then investigate the photonic bandgap (PBG) properties of the corresponding 2D Si posts and their tolerance to the structural deviation. We find that in the compound structure the overall PBGs are dominated by the sublattice with a higher symmetry, while the total symmetry is determined by the one with a lower symmetry. (C) 2008 American Institute of Physics. [DOI: 10.1063/1.3006061]
\end{abstract}

Photonic crystals (PCs) with periodically modulated refractive index are of interest for potential applications in large-scale optical integrated circuits. In comparison to the three-dimensional (3D) PCs, two-dimensional (2D) PC slabs offer efficient alternatives that can retain or approximate many of the desirable properties of 3D PCs and be mass producible over a large area with submicron resolution. ${ }^{1,2}$ The most widely studied 2D PCs are square and triangular lattices with isotropic circular-shaped unit cells. ${ }^{1-5}$ However, there have been comparatively few studies of 2D PCs with anisotropic unit cells except a recent work demonstrating control of the polarization modes of the emitted light. ${ }^{6}$

2D PC slabs are typically fabricated by conventional photolithography, electron beam lithography, and focused ion beam techniques, which require expensive tools and sometimes are only applicable to limited areas. Recently, we and others have demonstrated a simple yet robust method to produce orientationally modulated $2 \mathrm{D}$ patterns via pattern transformation triggered by elastomeric deformation..$^{7-9}$ Solvent-induced swelling instabilities of a single poly(dimethylsiloxane) (PDMS) membrane with a square lattice of micrometer-scale circular holes yield a library of 2D patterns with submicron resolution, including square- or rectangularlattices with orientationally modulated elliptical-shaped motifs (i.e., diamond plate), compound structures consisting of circles and elliptical lines, and a rectangular lattice of aligned ovals. ${ }^{8}$ By imposing an external elastic force that competes with internal stresses and manipulating the volume expansion ratio of the PDMS membrane using different swelling agents (e.g., organic solvents versus monomers), we can fine tune the structural details of the $2 \mathrm{D}$ patterns. The resulting patterns can then be used as etch masks to transfer the transformed patterns to semiconductors (e.g., Si), metals (e.g., $\mathrm{Au}$ ), and polymer films.

In this letter, we study the photonic bandgap (PBGs) of the corresponding 2D Si PCs as a function of the structural

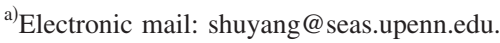

symmetries of the resulting patterns and their tolerance to structural deviation during fabrication. In a diamond plate structure with fatter ellipses, where the transformed pattern is imprinted from poly(ethylene glycol dimethacrylate) (PEGDMA), we find that the lowest transverse magnetic (TM) bandgap exhibits a high PBG quality, and in contrast to the square lattice with circular posts there are two additional gaps in TM modes. In a compound lattice with both sixfold and twofold rotational symmetries, we observe significantly different PBG properties from the rest of the structures, which is, however, close to that of a lattice with sixfold symmetry, and exhibits high quality TM gaps between the 2nd and 3rd bands and 10th and 11th bands, as well as an extra gap in the transverse electric (TE) modes.

The patterns with anisotropic unit cells studied in this letter are all transformed from a PDMS membrane with square lattice of circular holes [Fig. 1(a)]. When exposed to organic solvents (e.g., toluene) or liquid monomers (e.g., EGDMA), the PDMS membrane deforms into a "diamond plate" pattern with elliptical motifs mutually perpendicular to each other. The deformed pattern can be captured by photocuring of the EGDMA [Fig. 1(b)] or through application of $\mathrm{Fe}_{3} \mathrm{O}_{4}$ nanoparticles suspended in toluene on the PDMS membranes $^{8}$ [Fig. 1(c)]. Fatter elliptical features were obtained from the EGDMA pattern than from $\mathrm{Fe}_{3} \mathrm{O}_{4}$ nanoparticle assemblies because toluene produces a much larger volume expansion ratio, (as much as 130\%), ${ }^{10}$ thereby deforming the pores more than the EGDMA monomers. In addition, nanoscale line edge roughness can be observed from the nanoparticle film [see Figs. 1(c) and 1(d)], whereas that from PEGDMA appears much smoother [Fig. 1(b)].

By mechanically stretching the perforated PDMS membrane along a lattice direction, followed by swelling, we exert an external force favoring alignment along the strain direction to compete with the internal stresses caused by swelling. ${ }^{8}$ Figures 1 (d)-1(f) show the $\mathrm{Fe}_{3} \mathrm{O}_{4}$ nanoparticle assemblies lifted off from the stretched PDMS membranes. The patterns vary from a slightly distorted diamond plate to 


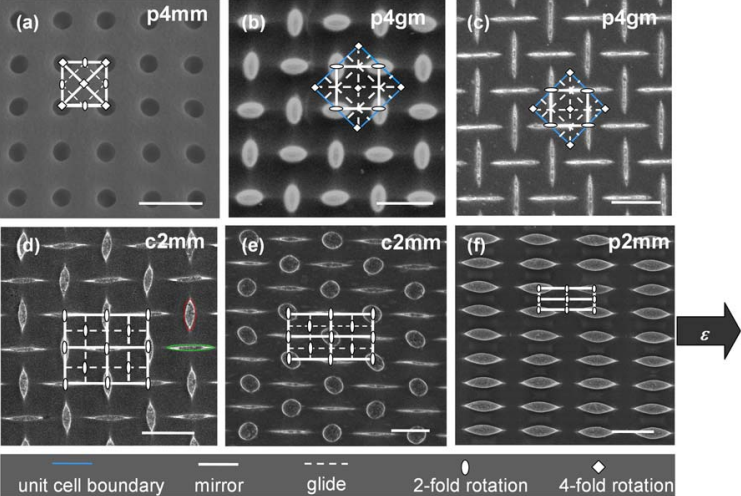

FIG. 1. (Color online) Illustration of the structural symmetries of 2D patterns obtained by elastic deformation of a PDMS membrane with a square lattice of circular holes. (a) SEM image of the original PDMS membrane with hole diameter $D=0.75 \mu \mathrm{m}$, pitch $P=1.5 \mu \mathrm{m}$, and depth $H=9 \mu \mathrm{m}$. [(b) and (c)] SEM images of "diamond plate" patterns imprinted from (b) PEGDMA and (c) $\mathrm{Fe}_{3} \mathrm{O}_{4}$ nanoparticle assemblies, respectively. (b) The EGDMA monomers mixed with 3 wt \% photoinitiator, Darocur 1173 (Ciba Specialty Chemicals Inc.), were applied to the PDMS membrane, which swelled the PDMS and deformed the holes into the diamond plate structure. The deformed pattern was captured by photo-cross-linking under UV light (dose of $\sim 4800 \mathrm{~mJ} / \mathrm{cm}^{2}$ ), followed by peeling off of the solidified film for characterization. (c) A solution of $\mathrm{Fe}_{3} \mathrm{O}_{4}$ nanoparticles suspended in toluene $(2.5 \% \mathrm{w} / \mathrm{v})$ is deposited onto the PDMS membrane. Nanoparticles convectively assembled on the PDMS membrane while it was swollen by toluene, capturing the deformed pattern. The nanoparticle film was then transferred to another substrate by microcontact printing for characterization. [(d)-(f)] SEM images of $\mathrm{Fe}_{3} \mathrm{O}_{4}$ nanoparticle assemblies from the stretched PDMS membranes at a uniaxial strain level of (d) $\varepsilon=15 \%$, (e) $\varepsilon=40 \%$, and (f) $\varepsilon$ $=80 \%$. Scale bars: $2 \mu \mathrm{m}$.

a compound lattice of circles and elliptical lines, to a rectangular lattice of aligned ovals at uniaxial strain levels of $\sim 15 \%, \sim 40 \%$, and $\sim 80 \%$, respectively. Furthermore, we can selectively oxidize the surface of the PDMS membrane to restrict the location for pattern transformation, thus generating more complex patterns with functional structures (see Fig. 2).

The nanoparticle assemblies or PEGDMA patterns can be conveniently transferred to photoresists, such as SU-8 (MicroChem Corp.), as etching masks to create 2D Si PC slabs. Detailed fabrication and characterization will be reported elsewhere. Here, we focus on theoretical investigation of the PBG properties of these transformed patterns as a function of structural symmetry.

The original PDMS membrane with circular pores arranged in a square lattice belongs to the plane group $\mathrm{p} 4 \mathrm{~mm}$
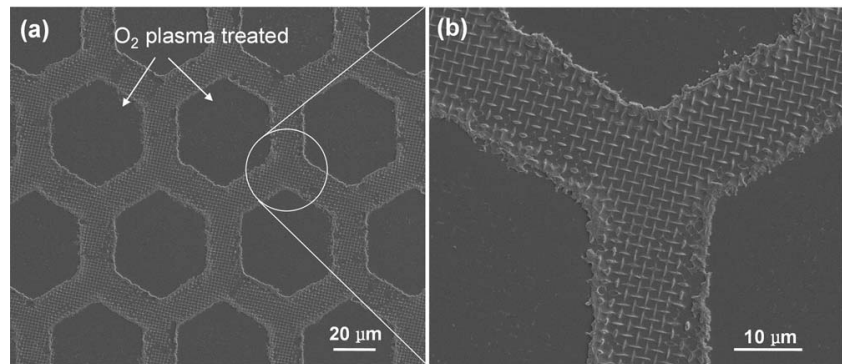

FIG. 2. SEM images of a honeycomb structure with localized diamond plate patterns. (a) Overview. (b) Close-up. The PDMS membrane was treated with oxygen plasma (PDC-001, Harrick Scientific Products, Inc.) at a power of $100 \mathrm{~W}$ for $30 \mathrm{~min}$ through a copper grid, followed by swelling with $\mathrm{Fe}_{3} \mathrm{O}_{4}$ nanoparticle/toluene solution. Since the oxidized region was not swollen by toluene, diamond plate pattern only appeared in the nonoxidized regions that were masked by the copper grid.
TABLE I. (Color online) Summary of PBG calculation results of Si $(\varepsilon$ $=13$ ) posts with various symmetries. $a=1.5 \mu \mathrm{m}$ and $c_{0}$ is the light velocity in vacuum. Inset: SEM images of epoxy pillar array replica molded from undeformed PDMS membrane, ${ }^{14}$ PEGDMA, and $\mathrm{Fe}_{3} \mathrm{O}_{4}$ nanoparticle assemblies imprinted from the deformed PDMS membranes, corresponding to those shown in Fig. 1. Scale bars: $2 \mu \mathrm{m}$.

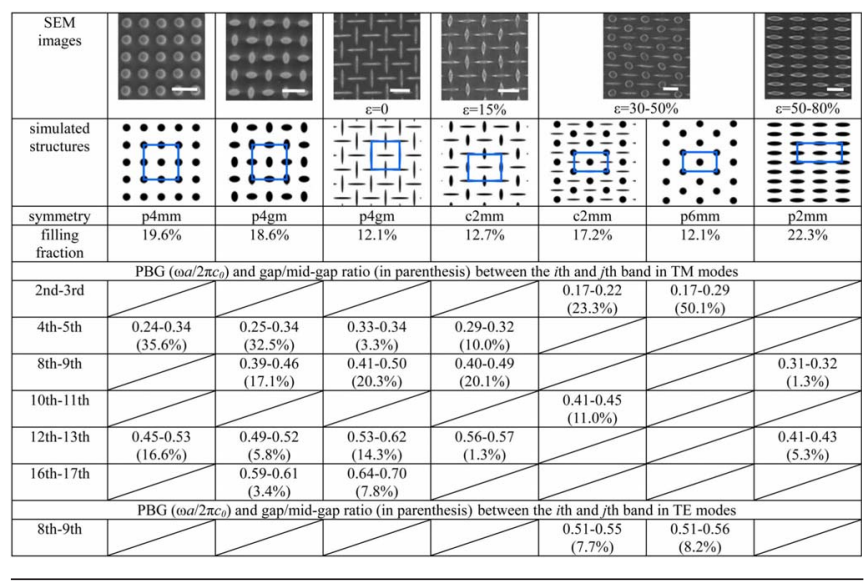

[Fig. 1(a)]. ${ }^{11}$ It transforms into a diamond plate pattern with p4gm symmetry [Figs. 1(b) and $1(\mathrm{c})] .{ }^{11}$ For the diamond plate pattern without strain, all elliptical motifs are identical with the same major and minor axes, and a spacing of $\sim 1.5 \mu \mathrm{m}$. When the perforated PDMS membrane is uniaxially stretched along a lattice direction, the square lattice changes to a rectangular one. At a low strain level $(\varepsilon$ $<30 \%$ ) the pattern is slightly distorted, changing its total symmetry to $c 2 \mathrm{~mm},{ }^{11}$ and the elliptical lines become thinner in the strain direction [Fig. 1(d)], whereas the ellipses perpendicular to the strain direction become fatter with the increase in the strain level. At $\varepsilon=30 \%-50 \%$, a binary pattern consisting of circles and degenerated flat ellipses is obtained [Fig. 1(e)]. The sublattice of circles belongs to the plane group $p 6 \mathrm{~mm}$, thus possessing a sixfold rotational symmetry. ${ }^{11}$ The total symmetry, however, is still determined by the sublattice of elliptical lines with a lower (twofold) symmetry. Hence, the compound structure remains to the plane group $c 2 \mathrm{~mm}$. When $\varepsilon>50 \%$, a rectangular lattice of ovals is obtained with structural symmetry of $p 2 \mathrm{~mm}$ [Fig. $1(\mathrm{f})]$.

The PBGs were calculated using the MIT photonic bandgap package ${ }^{12}$ from Si posts (dielectric constant, $\varepsilon_{r}=13$ ) with corresponding symmetries in $x y$ plane and an infinite height in $z$ direction surrounded by air. Both TE and TM modes were calculated from the 1st to the 20th band (see Table I). Since the transformed pattern has a periodic cell larger than the primitive cell of the original lattice on PDMS membrane, here, we consider a supercell consisting of $2 \times 2$ primitive cells for the square arrays of circular posts in the PBG calculation. Thus, the original bands are "folded" multiple times in the Brillouin zone, resulting in the appearance of bandgaps in higher bands than those using the primitive cell. The position and the width of corresponding PBGs, however, are not affected by the size of the supercell. The computational cells consisting of 4 "atoms" for each structure are marked in blue frames in Table I.

It is clear that most of the PBGs appear only in the TM modes since isolated $\mathrm{Si}$ posts are conducive to TM gaps, whereas a connected lattice favors TE gaps. ${ }^{13}$ For the square 
lattice with circular posts, there are two TM gaps: the lowest one between the 4th and 5 th bands with a quality factor $(\mathrm{QF})$ ( $\mathrm{QF}=$ gap/midgap ratio) of $35.6 \%$, and the other between the 12 th and 13th bands with a QF of $16.6 \%$. When the pattern is transformed into diamond plate structures, among them, the one obtained from PEGDMA with fatter ellipses possesses the highest QF, 32.5\% between the 4th and 5th TM bands, and two new gaps are opened in comparison to that from the circular posts. The diamond plate Si PCs transferred from nanoparticle assemblies with thinner elliptical lines have similar PBGs, except that the QF of the lowest TM gap decreases significantly to $3.3 \%$ due to the larger deformation of the PDMS membrane by toluene versus EGDMA monomer. The corresponding midgap positions also shift to higher frequencies since the filling fraction of high index material is decreased. Additional distortion by external force has no significant influence on PBG properties until the strain reaches a critical level, $30 \%-50 \%$, and a binary structure of circles and elliptical lines is generated. In the Si PC replicated from the binary pattern, we observe quite different PBG properties than those from diamond plates. Two TM gaps appear between the 2nd and 3rd bands and the 10th and 11th bands with QF of $23.3 \%$ and $11.0 \%$, respectively. Meanwhile, a TE band with QF of $7.7 \%$ between the 8th and 9th bands is also observed. In order to understand the origin of the unique PBG behavior, we investigate the PBGs of the sublattice of circles, which has $p 6 \mathrm{~mm}$ symmetry, and a supercell consisting of two circular-shaped cells (see Table I). The PBGs of the binary structure, except the TM gap between the 10th and 11th bands, are found close to but with lower quality than those of the sixfold sublattice, which can be attributed to the overall lowered symmetry. We believe that the filling fraction may also play an important role in the formation of the PBGs. The sublattice with sixfold rotational symmetry has a higher filling fraction of $\mathrm{Si}, 12.1 \%$, than the other one with twofold symmetry, $5.1 \%$. When $\varepsilon$ reaches $50 \%-80 \%$, the PBG quality is dramatically decreased and only two small TM gaps exist in the aligned oval structure. Nevertheless, the latter structures are of interest to control polarization modes of the electromagnetic field. ${ }^{6}$

In summary, we have developed an efficient method to fabricate a rich library of $2 \mathrm{D}$ photonic structures with anisotropic unit cells via harnessing elastomeric deformation of a single PDMS membrane. We study the PBG properties of the resulting 2D $\mathrm{Si}$ photonic structures as a function of their structural symmetries and their tolerance to the structural deviation. Among the transformed diamond plate patterns, the one imprinted from PEGDMA with fat ellipses displays four TM gaps between the 1st and 20th bands, and the highest predicted QF, 32.5\%. The compound structure with circles and elliptical lines, however, exhibits a PBG spectrum distinct from the rest of the structures and closer to that originating from sixfold symmetry. In this case, the sublattice with higher symmetry dominates PBG formation within a compound structure, and further work is needed to see if this is generally true. Understanding the relation of structural symmetry to PBG properties will help pave the way for rational design of efficient 2D PCs utilizing anisotropic unit cells.

This work is supported by the Office of Naval Research (ONR), Grant No. N00014-05-0303, and NSF/MRSEC, Grant No. DMR05-20020. The authors thank Jason Reed (Cornell University) for his assistance in some of the sample preparation.

${ }^{1}$ S. G. Johnson, S. H. Fan, P. R. Villeneuve, J. D. Joannopoulos, and L. A. Kolodziejski, Phys. Rev. B 60, 5751 (1999).

${ }^{2}$ E. Chow, S. Y. Lin, S. G. Johnson, P. R. Villeneuve, J. D. Joannopoulos, J. R. Wendt, G. A. Vawter, W. Zubrzycki, H. Hou, and A. Alleman, Nature (London) 407, 983 (2000).

${ }^{3}$ Y. Akahane, T. Asano, B. S. Song, and S. Noda, Nature (London) 425, 944 (2003).

${ }^{4}$ M. Loncar, D. Nedeljkovic, T. P. Pearsall, J. Vuckovic, A. Scherer, S. Kuchinsky, and D. C. Allan, Appl. Phys. Lett. 80, 1689 (2002).

${ }^{5}$ N. Carlsson, N. Ikeda, Y. Sugimoto, K. Asakawa, T. Takemori, Y. Katayama, N. Kawai, and K. Inoue, Opt. Quantum Electron. 34, 123 (2002).

${ }^{6}$ S. Noda, M. Yokoyama, M. Imada, A. Chutinan, and M. Mochizuki, Science 293, 1123 (2001).

${ }^{7}$ T. Mullin, S. Deschanel, K. Bertoldi, and M. C. Boyce, Phys. Rev. Lett. 99, 084301 (2007).

${ }^{8}$ Y. Zhang, E. A. Matsumoto, A. Peter, P. C. Lin, R. D. Kamien, and S. Yang, Nano Lett. 8, 1192 (2008).

${ }^{9}$ K. Bertoldi and M. C. Boyce, Phys. Rev. B 77, 052105 (2008).

${ }^{10}$ J. N. Lee, C. Park, and G. M. Whitesides, Anal. Chem. 75, 6544 (2003)

${ }^{11}$ International Tables for Crystallography, Space-Group Symmetry Vol. I, 5th ed. (Kluwer, Dordrecht, 2002).

${ }^{12}$ S. G. Johnson and J. D. Joannopoulos, Opt. Express 8, 173 (2001).

${ }^{13}$ J. D. Joannopoulos, R. D. Meade, and J. N. Winn, Photonic Crystals (Princeton University Press, Princeton, NJ, 1995).

${ }^{14}$ Y. Zhang, C. W. Lo, J. A. Taylor, and S. Yang, Langmuir 22, 8595 (2006) 